\title{
A perfect theory
}

\section{BOOK TITLE:}

The perfect theory: A century of geniuses and the battle over general relativity

\section{BOOK COVER:}

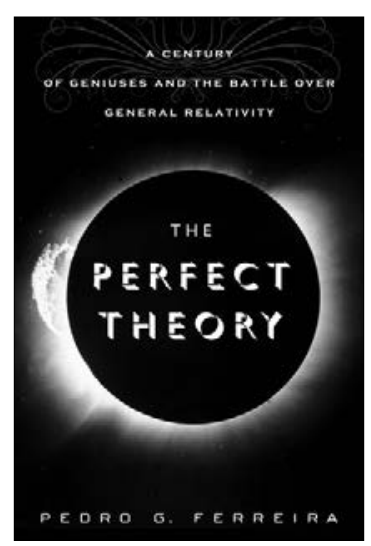

AUTHOR:

Pedro G. Ferreira

ISBN:

9780547554891 (hardcover)

\section{PUBLISHER:}

Houghton Mifflin Harcourt, Boston, MA; USD28

\section{PUBLISHED:}

2014

\section{REVIEWER:}

Peter Dunsby

EMAIL:

dunsby@gmail.com

\section{AFFILIATION:}

Department of Mathematics and Applied Mathematics, University of Cape Town, Cape Town, South Africa

\section{POSTAL ADDRESS:}

Department of Mathematics and Applied Mathematics, University of Cape Town, Private Bag X1, Rondebosch 7701, South Africa

\section{HOW TO CITE:}

Dunsby P. A perfect theory. S Afr J Sci. 2015;111(11/12), Art. \#a0129, 2 pages. http://dx.doi. org/10.17159/sajs.2015/a0129
Ask anyone on the street who Albert Einstein is or was and you will more often than not see their faces light up as they describe the eccentric man with crazy white hair and an infectious grin who changed our views about space and time, infinity and beyond - the very definition of genius. Karl Popper proclaimed that 'relativity' should be what all science should aspire to be. Like a work of art, Einstein's vision of the universe continues to fill us with wonder and delight. A reality filled with black holes and time warps, rapidly spinning neutron stars and gravitational waves, general relativity represents a magnificent unification of space and time, matter and energy, providing an explanation of some of nature's most profound mysteries and, as Pedro Ferreira describes it in his new book, very much 'The Perfect Theory'.

In The Perfect Theory, Professor Ferreira, a cosmologist at the University of Oxford tells the story of one of science's greatest accomplishments. He takes us from those early exploratory days of gedanken experiments, through the golden years of the late 1960s and 1970s when doing research in relativity was one of the trendiest things you could do as a theoretical physicist, all the way to the present day, when some are beginning to question its validity on the largest cosmological scales.

In the early 1900s, the scene was set for a scientific revolution. The clockwork deterministic world of Newton and Galileo, which had survived intact for more than 200 years, was about to be overturned by a 26 -year-old patent clerk named Albert Einstein. The problem related to the way objects from elementary particles to rocket ships moved relative to each other. According to Newtonian mechanics, speeds should add up in a straightforward way. If two cars moved towards each other, for example, the driver of one car would observe the other car to be moving at their speed plus the speed of the approaching car. The consensus at the time was that this fundamental law of addition should apply to everything, even particles of light called photons. Unfortunately, Maxwell's theory of electricity and magnetism painted a very different picture. The speed of light in a vacuum seemed to be the exception to this basic rule of addition. All observers, irrespective of their state of motion, should observe light to be travelling at the same speed. From 1887 to 1902, Michelson and Morley and others confirmed this exception experimentally, which led to a fundamental and irreconcilable mismatch between two of the major pillars of classical physics - a crisis only Einstein with his natural intuition, imagination and a pinch of common sense could resolve. How did he resolve it? Simply, by keeping the speed of light constant and determining what the consequence would be for the Newtonian equations of motion. It seems almost obvious now, when one looks back, but the result of making this rather simple change to the framework of physics was revolutionary. Not only did it unify mechanics and electromagnetism, it banished the Galilean notions of absolute space and time to the dustbin of history.

It is worth mentioning at this point that Einstein's 1905 paper on special relativity was one of four remarkable pieces of work that shook the world of physics that year. One explained how to measure the size of molecules in a liquid, the second determined their movement - Brownian motion - and a third described how quanta of light could knock out electrons in metals (the photoelectric effect became the basis of quantum mechanics and electrochemistry). It was this idea, not special (or general) relativity, that eventually won him the Nobel Prize. At this point, most mortals would have put down their pen and taken a well-deserved break, but not Einstein. A few months later, he pointed out in a fifth paper that matter and energy are interchangeable at the atomic level through $E=m c^{2}$, arguably the most famous equation in history, providing the scientific foundations of the nuclear age. And with that, Einstein's 'miracle year' came to an end.

Einstein and others now turned their attention to the problem of how to make gravity fit into this new spacetime description of nature. As Ferreira explains, Einstein's real genius lay in his ability to take well-known observations about the way the universe worked and then use his imagination to construct outlandish thought experiments which revealed deep physical insight and concrete testable predictions. It was Galileo's fabled Pisa experiment which lay the foundations of Einstein's famous lift experiments. The fact that balls of different masses fell to the ground at the same rate implied that gravity was just another inertial effect and that experiments performed in small regions of spacetime should be indistinguishable from what would be obtained in special relativity (in the absence of gravity). This equivalence of gravity and acceleration was the critical ingredient for building a theory of gravity which included special relativity as a local limit. The lift experiments also pointed to some key predictions of this more general theory. For example, imagine accelerating a lift in empty space and observing the motion of a light ray within. The light ray would appear to curve down. Now magically transport the lift to the surface of the earth. The equivalence of acceleration and gravity would imply that the light ray would also bend in the earth's gravitational field. Without any equations, Einstein was able to predict gravitational lensing, a powerful method now used to constrain cosmological models. Finally, take the lift and throw it down a mine shaft. Two particles initially at rest appear to move towards each other, suggesting that gravity is described by spacetime curvature, so do not move on lines that remain parallel.

Thought experiments are one thing, but converting these visualisations into a set of precise equations is another matter. With help from Marcel Grossman, Einstein was able to build a mathematical framework based on Riemannian geometry able to describe how mass and energy distort the fabric of spacetime and how particles move on curved spacetime. Ferreira compares this undertaking to 'learning Sanskrit from scratch and then writing a novel in it'. With the gravitational field equations in the bag, general relativity could be used to determine the correct orbit of Mercury, which Newton's theory of gravity never quite got right because it did not take into account the curvature of spacetime. A paradigm shift in the understanding of gravity and how it affected the cosmos had occurred.

With general relativity largely accepted by the physics community and Einstein now one of the most famous men in the world, the exciting quest to discover its consequences started in earnest. From the trenches of the 
First World War, Karl Schwarzschild discovered an exact solution of the field equations describing the spacetime around spherically symmetrical objects such as stars and the cores of galaxies. This discovery provided a way of determining the correct motion of planets around our sun, and how light moves on the curvature of spacetime - a key step in the development of the theory of gravitational lensing which is used today to map the distribution of dark matter. Even in weakly gravitating systems like the earth, this solution is required to calculate the precise orbits of GPS satellites. You see, general relativity is even important for navigating through cities using our smartphones!

The discovery of the Schwarzschild solution also represented the beginning of an exploration of the most exotic objects in the universe - black holes. The idea that the end state of stars could lead to a singular point, at which gravitational tidal forces become infinite and the laws of physics break down, was one of the most radical consequences of Einstein's theory. Even more mysterious was the fact that singularity was hidden from the rest of the universe by a surface (called the horizon) from which not even light could escape. It took decades of research before a complete understanding of this solution was obtained and even now there is still controversy over the exact nature of the surface that hides the singularity from prying eyes.

During the late 1960s and 1970s, Einstein's description of nature's most mysterious force reached maturity. By now numerous exact solutions had been found to describe objects from wormholes to cosmic strings and even the universe itself. However, it was on the smallest possible scales, where the determinacy breaks down and the mysterious quantum world rules supreme, that general relativity finally met its match. It seems that despite decades of searching for a self-consistent theory of quantum gravity, we are still a very long way from describing what happened at the moment of the Big Bang itself.

If this is not troubling enough, even the classical theory might have issues on the largest possible scales, when one deals with the universe as a whole. In 1998, our view of the expansion history of the universe was fundamentally changed - a result which rocked the cosmology community to its core. Two international groups of astrophysicists collected data on the light output from distant stellar explosions called type la Supernovae in order to measure how the universe has expanded over its 13.7-billion-year lifetime. Based on the simplest cosmological model at the time, which they originally had proposed, Einstein and de Sitter expected that the expansion would be slowing down as galaxies pulled toward each other as a result of their gravitational attraction. What they found was quite remarkable and such a surprise that initially both teams did not believe their results.

The interpretation of these observations was startling. Firstly, the Einstein-de Sitter model was completely ruled out, and secondly, if the geometry of the universe was still taken to have Friedmann-RobertonWalker form, an almost preposterous conclusion resulted: the universe has an accelerated expansion today! Now, the problem is that ordinary matter cannot do this, so some new form of matter with negative pressure (known as dark energy) must be dominating the expansion history today. The nature of dark energy is without doubt the most puzzling mystery in cosmology and astrophysics today, and finding an explanation to what it is (or is not) is almost certainly the Holy Grail of theoretical cosmology. Part of the mystery is that we really do not have any clue what it is or whether we will even be able to find an answer to this deep fundamental question. New physics, or a change in how gravity behaves, might be required to resolve this impasse.

The last chapter of Ferreira's book, 'Something is going to happen', brings us almost full circle to the beginning of this story. Like then, the time is ripe for the next great revolution in physics. Only time will tell, but future developments in gravitational physics and cosmology might lead to an explanation of what the dark side of the universe is, whether the universe ends in a cataclysmic Big Rip or in frozen isolation, and whether we might again be forced to change the fundamental laws of physics. It is even possible that 'The Perfect Theory' might not be so perfect after all. 